\title{
Polariton effects in the dielectric function of ZnO excitons obtained by ellipsometry
}

Cite as: Appl. Phys. Lett. 96, 031904 (2010); https://doi.org/10.1063/1.3284656

Submitted: 22 October 2009 . Accepted: 12 December 2009 . Published Online: 19 January 2010

Munise Cobet, Christoph Cobet, Markus R. Wagner, Norbert Esser, Christian Thomsen, and Axel Hoffmann

\section{ARTICLES YOU MAY BE INTERESTED IN}

\section{A comprehensive review of $\mathrm{ZnO}$ materials and devices}

Journal of Applied Physics 98, 041301 (2005); https://doi.org/10.1063/1.1992666

Infrared dielectric functions and phonon modes of high-quality ZnO films

Journal of Applied Physics 93, 126 (2003); https://doi.org/10.1063/1.1526935

Determination of the optical constants of zinc oxide thin films by spectroscopic ellipsometry

Applied Physics Letters 72, 3261 (1998); https://doi.org/10.1063/1.121617

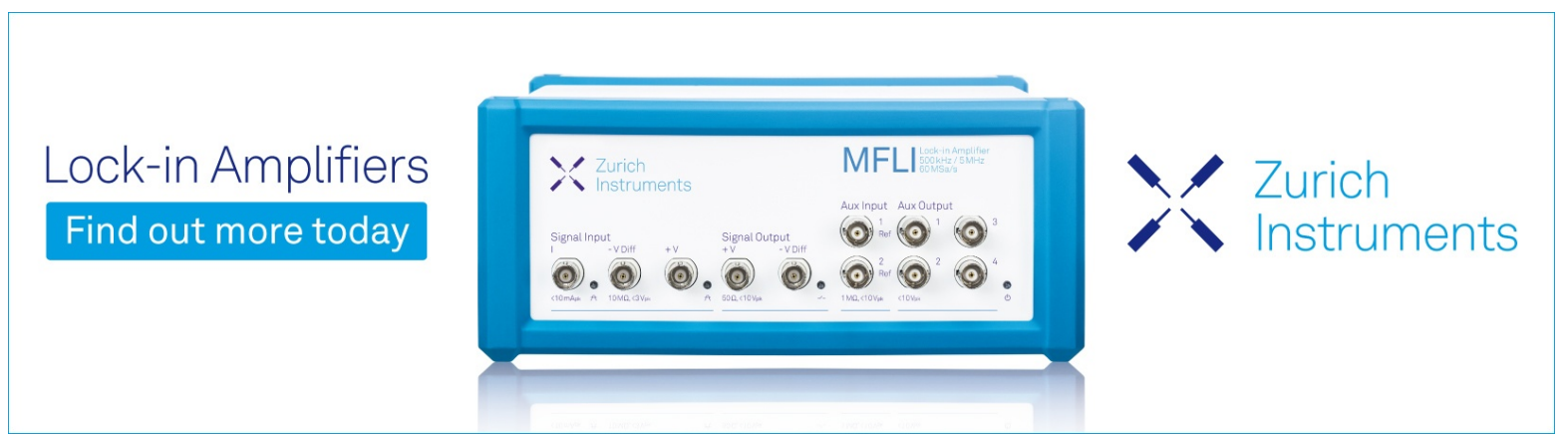




\title{
Polariton effects in the dielectric function of $\mathrm{ZnO}$ excitons obtained by ellipsometry
}

\author{
Munise Cobet, ${ }^{1, a)}$ Christoph Cobet, ${ }^{2}$ Markus R. Wagner, ${ }^{1}$ Norbert Esser, ${ }^{2}$ \\ Christian Thomsen, ${ }^{1}$ and Axel Hoffmann ${ }^{1}$ \\ ${ }^{1}$ Institute for Solid State Physics, Technische Universität Berlin, 10623 Berlin, Germany \\ ${ }^{2}$ ISAS-Institute for Analytical Sciences, 12489 Berlin, Germany
}

(Received 22 October 2009; accepted 12 December 2009; published online 19 January 2010)

\begin{abstract}
The complex dielectric tensor of $\mathrm{ZnO}$ in the regime of the excitonic transitions is determined with ellipsometry and analyzed concerning the quantization of the electromagnetic field in terms of coupled polariton-eigenmodes. Negative sections in the real part indicate the significant formation of polaritons for the dipole-allowed excitons of the three upper valence-bands $\Gamma_{7}, \Gamma_{9}, \Gamma_{7}$. The transverse-longitudinal splittings which separate the upper polariton branch from the lower branch, corresponding to the $\mathbf{k}$-vector of the used light, are deduced precisely for each subband. Mainly for $\mathbf{E} \| c$, additional absorption peaks are observed at the longitudinal B-exciton and closely above. One is considered to be a mixed-mode and the other is seen as a consequence of interference effects in an exciton free surface layer which is also visible in reflectance anisotropy spectroscopy. (C) 2010 American Institute of Physics. [doi:10.1063/1.3284656]
\end{abstract}

Apart from the potential use of $\mathrm{ZnO}$ for ultraviolet light emitting diodes, photodetectors or transparent conductors for solar cells, the strong excitonic binding energy $\left(\mathrm{E}_{\mathrm{b}}\right.$ $\approx 60 \mathrm{meV}$ ) in combination with the polar bonding character exhibits many more interesting phenomena. The interaction with an electromagnetic wave comes along with strong polarization fields traveling through the material in terms of coupled transverse and longitudinal eigenmodes. Since the mixed state of the radiation field and polarization waves can be considered as bosonic quasiparticles, a collective condensation in the lowest quantum state (quasi Bose-Einstein condensation) at $\mathbf{k}=0$ is possible and already impressively shown by resonantly exciting them in a microcavity. ${ }^{1}$ Using this cavity polariton condensation, polariton lasing is one of the most fascinating applications because of the extremely low threshold power operation without the need of population inversion. The wide-band gap semiconductors are favored since the strong coupling regime and the coherence state is stable at room temperature and above. Recently, GaN-based polariton lasing at room temperature has been realized. ${ }^{2}$ Using $\mathrm{ZnO}$ as active medium, further enhancement is predicted in operating temperature and Rabi-splitting between the different modes. ${ }^{3}$ For a development of these devices, ${ }^{4}$ the precise knowledge of the polariton eigenstates is crucial to optimize the resonator structure. The dispersion relationship for excitonic polaritons is represented by the dipole allowed (optical) eigenstates as a function of the wave vector $\mathbf{k}$ which basically depends on the direction of light propagation in the material regarding the crystallographic directions. ${ }^{5}$

The optical properties, e.g., specular reflection and absorption are governed by the index of refraction $n$ and the extinction coefficient $\kappa$ which are known as frequency dependent quantities that define the dielectric function (DF).

The group symmetry of wurtzite $\mathrm{ZnO}$ is $C_{6 v}$. For a complete description of the linear optical response of this uniaxial anisotropic crystal, two independent components of the DF referring to electronic excitation parallel $\left(\varepsilon_{\|}\right)$and per-

${ }^{a)}$ Electronic mail: rakel@physik.tu-berlin.de. pendicular $\left(\varepsilon_{\perp}\right)$ to the optical $c$-axis have to be probed.

Ellipsometry is the method of choice because it allows a direct and reference-free determination of the whole set of linear optical functions by measuring the amplitude ratio $\Psi$ of and the phase shift $\Delta$ between parallel and perpendicular polarized parts of the reflected light beam. The real and imaginary part of the DF are determined in a straightforward manner and approximations in a subsequent KramersKronig analysis are avoided. Most of the previous work is based on either reflectivity or absorption/transmission measurements and always regarded separately without any direct association. Gaining a complete picture of the correlation between the reflectivity and the dielectric real and imaginary part is strongly desirable.

In this letter we present ellipsometric measurements of the fine structure of the $\mathrm{A}, \mathrm{B}$, and $\mathrm{C}$-excitons in $\mathrm{ZnO}$ including a determination of the longitudinal-transverse splittings $\Delta_{\mathrm{LT}}$ of their individual polaritons under a certain angle of incidence. All previously published ellipsometric results on $\mathrm{ZnO}$, e.g., ${ }^{6,7}$ could not resolve the energetic positions of $\mathrm{A}$ and B. Possible reasons can be either an overlayer of condensed water on the surface, if cooling in a non-UHV environment, which causes a considerable damping of structures, or from an insufficient resolution of the monochromator.

We used an $a$-plane bulk crystal grown by the hydrothermal method with a surface normal along the $[11 \overline{2} 0]$ offered by CrysTec. The XRD FWHM-value of the rocking curve using the (1120)-reflection at $\omega=28.17^{\circ}$ is 135.9 arcsec and the Raman shift of the $E_{2}$ (high) mode is recorded to be $437.4 \pm 0.4 \mathrm{~cm}^{-1}$, respectively. The values indicate a rather good crystal quality (for an $a$-plane substrate) and a small build-in compressive strain. ${ }^{8}$ However, this sample was chosen to be a representative material. Other bulk samples and homoepitaxial epilayers were also investigated but in this letter we do not attempt to evaluate the observed differences in the absolute values of the split subbands A and B, which relate to different internal strain levels. All measurements were performed with a rotating analyzer ellipsometer with an angle of incidence of $67^{\circ}$ by using a normal incidence mono- 
chromator $3 \mathrm{mNIM}$ at BESSY II. The base pressure was kept at $3 \times 10^{-10}$ mbar. The resolution of the monochromator $E / \Delta E$ is better than 10000 and spectroscopic measurements were taken with intervals of $50 \mu \mathrm{eV}$. The polarization vector of the beam was tilted to $20^{\circ}$ to the plane of incidence. The $a$-plane samples were oriented with the $c$-axis perpendicular or parallel to the plane of incidence, so that the p-polarized electric field component is $\mathbf{E}_{\mathrm{p}} \perp c$ and $\mathbf{E}_{\mathrm{p}} \| c$. In this case, the tensor components can be separated more readily with a two

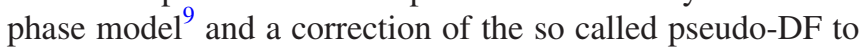
the ordinary and extraordinary DF $\varepsilon_{\perp}$ and $\varepsilon_{\|}$was processed by using the Fresnel formulas for uniaxial anisotropic media. ${ }^{10}$ Reflectance anisotropy spectroscopy (RAS) was performed with a home build setup in normal incidence configuration.

For the detailed description of the optical response functions closely below the band gap we consider the three exciton series which are derived from a $\mathrm{Zn} 4 s$-like conduction band with $\Gamma_{7}$ symmetry and an $\mathrm{O} 2 p$-like valence band. $\mathrm{Ne}$ glecting spin, this is split into two bands of $\Gamma_{5}$ and $\Gamma_{1}$ symmetry by the hexagonal crystal field. The relativistic spinorbit coupling lifts the degeneracy of the $\Gamma_{5}$ into an upper $\Gamma_{9}$ and a lowered $\Gamma_{7}$, while the $\Gamma_{1}$ becomes the lowest $\Gamma_{7}$. In $\mathrm{ZnO}$, the spin-orbit splitting $\Delta_{s o}$ is most commonly assumed to be negative due to the narrow $\mathrm{Zn} 3 d$ level, ${ }^{11}$ which is actually a closed shell core level. This enforces an overlap of their wave functions which results in a strong $p$ - $d$ repulsion. A reversal in the two upper valence states $\Gamma_{7}$ and $\Gamma_{9}$ is considered as a consequence. We prefer to use the reversed ordering, since magnetophotoluminescence on the hole-state related fine-splitting of bound excitons ${ }^{12}$ strongly support this assumption. The three ground state exciton bands $(n=1)$ are labeled A, B, and C in order of increasing energy. Each band is twofold degenerated for $\mathbf{k}=0$.

The ellipsometric spectra of $\mathrm{ZnO}$ in the vicinity of the $\mathrm{A} \Gamma_{7}, \mathrm{~B} \Gamma_{9}$, and $\mathrm{C} \Gamma_{7}$ at $\mathrm{T}=10 \mathrm{~K}$ is given in Figs. 1(a) and 1(c) together with (d) the RAS data and (e) the dispersion in $\mathbf{k}$-space calculated from Eq. (1). For an analysis it is very important to regard the interdependent relation of the real and imaginary part at distinct structures, whereas the imaginary part is directly proportional to the absorption. Because of $\nabla \varepsilon_{0} \varepsilon(\omega) \mathbf{E}=0$ and $\mathbf{E}_{0} \times \mathbf{k}^{i(\mathbf{k} r-\omega t)}=0 \Rightarrow \mathbf{E} \perp \mathbf{k}$, where $\varepsilon_{0}$ and $\varepsilon(\omega)$ are the dielectric constants in vacuum and matter, $\mathbf{E}$ is the electric-field vector of the electromagnetic wave with a frequency $\omega$ and $\mathbf{k}$ is the wave vector, the principal solution for light in matter (and vacuum) is a transverse electromagnetic wave. The other solution $\varepsilon(\omega)=0$, which is only valid in matter, gives the resonance for a longitudinal mode $\mathbf{E}\left(\omega_{\mathrm{L}}\right) \| \mathbf{k}$ with antiparallel polarization and electric field $\varepsilon_{0} \mathbf{E}=-\mathbf{P}$. Because of the noncrossing rule in the region of the stop-band between $\omega_{0}$ and $\omega_{\mathrm{L}}$ there is only very small fraction of light propagating the sample and the penetration depth is shorter than the wavelength. We identify the transverse resonance energies $\hbar \omega_{0}$ by the local maxima in the imaginary part $\varepsilon_{2}$ and the longitudinal resonance $\hbar \omega_{\mathrm{L}}$ by the zero crossing point toward positive values (the high energy end of the negative region) of the real part $\varepsilon_{1}$ corresponding to the Lorentz oscillator model. This allows a direct and very precise determination of both resonances $\omega_{0}$ and $\omega_{\mathrm{L}}$ and the transverse-longitudinal splitting $\Delta_{\mathrm{LT}}$. By contrast, in reflectivity measurements [see Fig. 1(c)] where finite damping causes always a considerable broadening in the spectral

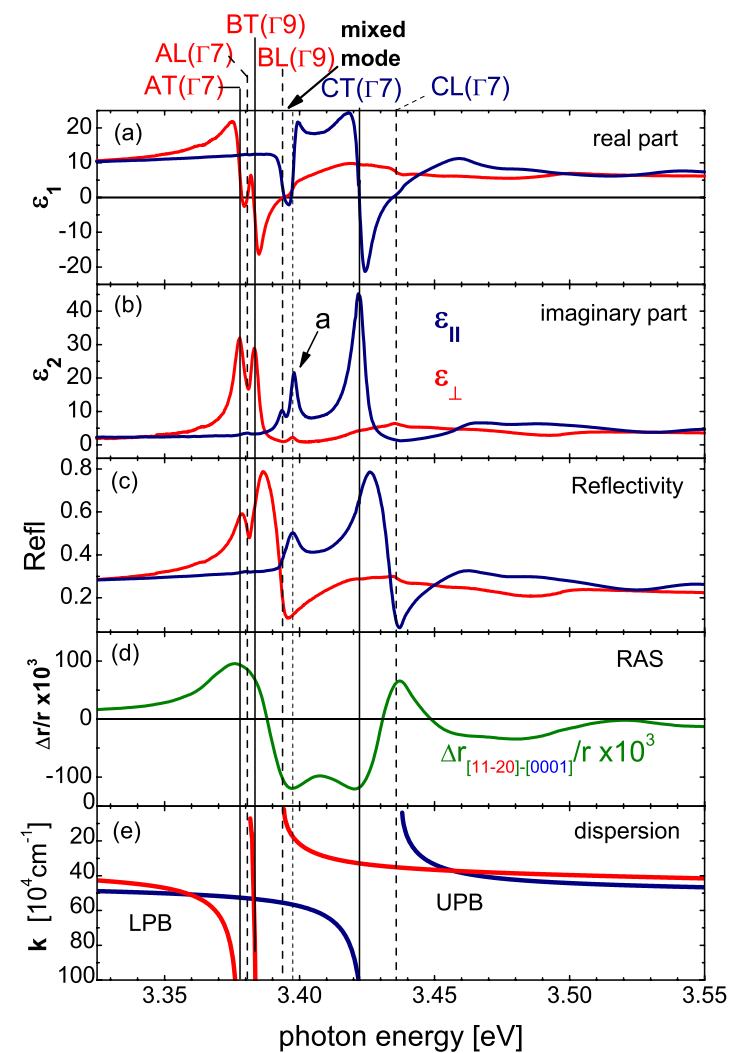

FIG. 1. (Color online) (a) Real and (b) Imaginary part of the DF and (c) Reflectivity obtained from ellipsometry, (d) RAS and (e) Dispersion relation for $n=1$ exciton-polaritons. The transversal (longitudinal) energies are marked with solid (dashed) lines. The anomaly "a" is marked with an arrow. The RAS data resemble the peaks for $\mathbf{E} \perp \mathrm{c}$ with positive values while the peaks in $\mathbf{E} \| \mathrm{c}$ are negative.

width of the stop-band, the determination of $\omega_{0}$ can only be obtained by a very complicated line shape analysis.

The k-dependence of our data in Fig. 1(e) was calculated for $\mathbf{k} \perp c$ by using the so-called polariton equation and the following assumption: ${ }^{13}$

$$
\mathbf{k}^{2}=\frac{n^{2} \omega^{2}}{c^{2}} ; n^{2}=\varepsilon(\omega)=\varepsilon_{\mathrm{b}} \times \frac{\omega_{\mathrm{L}}^{2}-\omega^{2}}{\omega_{0}^{2}-\omega^{2}},
$$

where $\omega_{\mathrm{L}}$ and $\omega_{0}$ are the longitudinal and transverse resonance frequencies and $\varepsilon_{\mathrm{b}}$ is the background dielectric constant, respectively. We obtain a $\varepsilon_{\mathrm{b}}^{\perp}$ of 6.69 and $\varepsilon_{b}^{\|}$of 7.47 for the excitonic region from our spectra. The longitudinal wave solution at $\mathbf{k}=0$ marks also the onset of the upper polariton branch (UPB), going over into a photonlike dispersion, while the lower polariton branch becomes excitonlike within the bottleneck-region. The strong interaction of very close lying oscillators, namely the $\mathrm{A}$ and $\mathrm{B}$ excitons in $\mathrm{ZnO}$, has to be taken into account by a superposition of their resonances and shows up in a considerable distortion of the polariton branches as displayed in Fig. 1(e). This explains the fact, that the LT-splitting is strongly reduced for the A exciton, while it is increased for the B exciton-polariton (see Table I). It is therefore not possible to deduce the oscillator strength by these splittings correctly which should actually both have the same value. $^{14}$

The ordinary tensor component $\varepsilon_{\perp}$ was obtained by an ellipsometric measurement with $\mathbf{k}$ in the $x y$-plane (normal to the $c$-axis), therefore oscillators with $\Gamma_{5}$ symmetry $\left(\mathrm{A} \Gamma_{7}\right.$ and $\left.\mathrm{B} \Gamma_{9}\right)$ oriented along $x$ and $y$ are excited. We find a nonde- 
TABLE I. Exciton-polariton parameters in $\mathrm{ZnO}$ obtained from ellipsometry (in electron-volt).

\begin{tabular}{lccccc}
\hline \hline \multicolumn{1}{c}{ Exciton } & Polarization & $\hbar \omega_{0}$ & $\hbar \omega_{\mathrm{L}}$ & $\Delta_{\mathrm{LT}}$ & $\mathrm{n}=2$ \\
\hline $\mathrm{A}_{7}$ & $\perp$ & 3.3778 & 3.3805 & 0.0027 & 3.4214 \\
$\mathrm{~B}_{9}$ & $\perp$ & 3.3834 & 3.3940 & 0.0106 & 3.4276 \\
$\mathrm{C}_{7}$ & $\|$ & 3.4224 & 3.4345 & 0.0121 & 3.4630 \\
Mixed mode & $\|($ weak $\perp)$ & 3.3934 & 3.3977 & 0.0043 & $\cdots$ \\
Anomaly & $\|($ weak $\perp)$ & $\cdots$ & 3.3979 & $\cdots$ & $\cdots$ \\
\hline \hline
\end{tabular}

generate transverse and a longitudinal branch for $\mathbf{E} \| y$ and $\mathbf{E} \| x$, respectively. The extraordinary tensor component $\varepsilon_{\|}$ was measured with the E-field vector oriented $20^{\circ}$ to the plane containing both the wave vector and the $c$-axis. The external angle between $\mathbf{k}$ and $c$ is $23^{\circ}$, hence $\mathbf{k}$ can be divided into two components. For the one component $\mathbf{k} \| c$, only pure transverse modes are expected, while for the other component $\mathbf{k} \perp c$ and $\mathrm{E} \| c$ only longitudinal excitons are allowed. For angles in between, mixed-modes will appear, which have components of both transverse and longitudinal polarizations. ${ }^{15}$ As a result, the extraordinary polariton $\mathrm{C} \Gamma_{1}$, which couples only to $\mathbf{E} \| c$, and a mixed mode polariton is observed. For the mixed mode, the absorption peak for the transverse eigenmode $\omega_{0}^{\text {mix }}$ exactly matches $\omega_{\mathrm{L}}^{B}$ of the ordinary part. The value of $\omega_{\mathrm{L}}^{\text {mix }}$ is again obtained by $\varepsilon_{\|}=0$. The LT-splitting of the mixed mode is $4.3 \mathrm{meV}$. From $\Delta_{\mathrm{LT}}(\phi)$ $=\Delta_{\mathrm{LT}}\left(0^{\circ}\right) \cos ^{2} \phi$, however, this could imply that the actual LT-splitting of the B exciton-polariton should be around 5.2 $\mathrm{meV}$ without level repulsion of the A-exciton. Regarding the additional absorption structure at $\omega_{\mathrm{L}}^{\text {mix }}$ in $\varepsilon_{2}$ it is obvious that this is not consistent with the corresponding real part $\varepsilon_{1}$ when applying the Lorentz oscillator model and KramersKronig relations. Owing to the effort of Hopfield, Thomas, Pekar, and Lagois ${ }^{5,16,17}$ there exists already a quite good understanding of the experimental findings of anomalous peaks by the involve of an exciton free surface layer ("dead layer") in connection with additional boundary conditions (ABC) for the Maxwell equations as a consequence of spatial dispersion. We believe that this anomalous peak relates to multiple reflection interferences, which takes place in the "dead layer," which may be thicker than the pure Bohr radius due to surface imperfections. Also, small misalignments of the $c$-axis can result in slight admixtures of states and the weak appearance of the mixed mode and $\mathrm{C}_{\mathrm{L}} \Gamma_{7}$ in $\varepsilon_{\perp}$ and $\mathrm{B}_{\mathrm{L}} \Gamma_{9}$ in $\varepsilon_{\|}$is seen as a consequence. Narrow anomalous spikes due to isotropic points were also reported ${ }^{18}$ and explained by an energy transfer from one polarization to another. To address this problem, we performed RAS-measurements in the spectral region of interest [Fig. 1(d)]. The crosschecking with RAS is very useful to verify ellipsometric data for anisotropic crystals. ${ }^{19}$ The RAS gives the amount of anisotropy by the ratio of the difference of reflectivity parallel and perpendicular $\Delta r_{[11 \overline{2} 0]-[0001]}$ and the total reflectivity. At isotropic points of the optical functions, where $\varepsilon_{\perp}$ and $\varepsilon_{\|}$are equal, the RAS signal is zero. It is assumed, that a residual surface induced anisotropy (e.g., reconstructions) is absent or negligible. The RAS measurements were performed at almost normal incidence, therefore the mixed mode is expected to disappear. Indeed the mixed branch vanishes but the anomaly does not. It again does not coincide with the isotropic points. It has to be noticed, that the RAS spectrum was recorded at
$\mathrm{T}=120 \mathrm{~K}$, hence the $\mathrm{A}$ and $\mathrm{B}$ structures are not resolved and the corresponding structures are subsequently shifted about $9 \mathrm{meV}$ to higher energies.

In summary, the presented study shows the possibility of precise determination of the LT-splitting for every individual exciton-polariton with ellipsometry for a certain angle of incidence. The most interesting features found in the spectra are the negative sections in $\varepsilon_{1}$ and anomalous absorption peaks in $\varepsilon_{2}$. From the classical Lorentz oscillator model we identify the longitudinal eigenfrequency usually by a vanishing $\varepsilon$ and the transverse by maxima in the absorption, but the anomalous peak closely above $\mathrm{B}_{\mathrm{L}}$ does not satisfy to this description. This peak correlates to the zero crossing of $\varepsilon_{1}$ of a mixed mode. Within our observations, it turns out that one cannot neglect the effects of strong coupling when using ellipsometry and RAS in the spectral region of strong excitonic absorption. The suggested description of optical response only manipulates the "effective" optical parameters, therefore additional structures near excitonic resonances in the spectra do not reduce the utility of Kramers-Kronig consistence. The next step is to verify the "dead layer" assumption with a suitable model fit by taking $\mathrm{ABC}$ explicitly into account.

We wish to acknowledge the supply of $\mathrm{ZnO}$ samples by B. Meyer and N. H. Nickel and useful discussions with R. Goldhahn. We are also grateful to Walter Braun of the BESSY GmbH for always kindly supporting our work. This work was supported by the European Community through the FP7-NanoCharM project.

${ }^{1}$ J. Kasprzak, M. Richard, S. Kundermann, A. Baas, P. Jeambrun, J. M. J. Keeling, F.M. Marchetti, M.H. Szymanska, R. Andre, J.L. Staehli, V Savona, P.B. Littlewood, B. Deveaud, and L. S. Dang, Nature (London) 443, 409 (2006).

${ }^{2}$ S. Christopoulos, G. B. Höger von Högersthal, A. J. D. Grundy, P. G. Lagoudakis, A. V. Kavokin, J. J. Baumberg, G. Christmann, R. Butte, E. Feltin, J.-F. Carlin, and N. Grandjean, Phys. Rev. Lett. 98, 126405 (2007). ${ }^{3}$ S. Faure, T. Guillet, P. Levebvre, T. Bretagnon, and B. Gil, Phys. Rev. B 78, 235323 (2008).

${ }^{4}$ C. Sturm, H. Hilmer, R. Schmidt-Grund, C. Czekalla, J. Sellmann, J. Lenzner, M. Lorenz, and M. Grundmann, J. Vac. Sci. Technol. B 27, 1726 (2009).

${ }^{5}$ J. Lagois and K. Hümmer; Phys. Status Solidi B 72, 393 (1975).

${ }^{6}$ R. Schmidt-Grund, M. Schubert, B. Rheinländer, D. Fritsch, H. Schmidt, E. M. Kaidashev, M. Lorenz, C. M. Herzinger, and M. Grundmann, Thin Solid Films 455, 500 (2004).

${ }^{7}$ G. E. Jellison, Jr. and L. A. Boatner, Phys. Rev. B 58, 3586 (1998).

${ }^{8}$ J. M. Calleja and M. Cardona, Phys. Rev. B 16, 3753 (1977).

${ }^{9}$ D. E. Aspnes, J. Opt. Soc. Am. 70, 1275 (1980).

${ }^{10}$ R. M. A. Azzam and N. B. Bashara, Ellipsometry and Polarized Light (North-Holland Personal Library, Amsterdam, 1987).

${ }^{11}$ W. R. L. Lambrecht, A. V. Rodina, S. Limpijumnong, B. Segall, and B. K. Meyer, Phys. Rev. B 65, 075207 (2002).

${ }^{12}$ M. R. Wagner, J.-H. Schulze, R. Kirste, M. Cobet, A. Hoffmann, C. Rauch, A. V. Rodina, B. K. Meyer, U. Röder, and K. Thonke, Phys. Rev. B 80, 205203 (2009).

${ }^{13}$ P. Y. Yu and M. Cardona, Fundamentals of Semiconductors (Springer, Berlin, 2001).

${ }^{14}$ C. Klingshirn, Semiconductor Optics (Springer, Berlin, 2005).

${ }^{15}$ J. J. Hopfield and D. G. Thomas, J. Phys. Chem. Solids 12, 276 (1960).

${ }^{16}$ J. J. Hopfield and D. G. Thomas, Phys. Rev. 132, 563 (1963).

${ }^{17}$ S. J. Pekar, Sov. Phys. Solid State 4, 953 (1962).

${ }^{18}$ G. Blattner, G. Kurtze, G. Schmieder, and C. Klingshirn, Phys. Rev. B 25 , 7413 (1982)

${ }^{19}$ U. Rossow, R. Goldhahn, D. Fuhrmann, and A. Hangleiter, Phys. Status Solidi B 242, 2617 (2005). 